(C) (C) 2021 IEEE. Personal use of this material is permitted. Permission from IEEE must be obtained for all other uses, in any current or future media, including reprinting/republishing this material for advertising or promotional purposes, creating new collective works, for resale or redistribution to servers or lists, or reuse of any copyrighted component of this work in other works. 


\title{
Energy shaping control of hydraulic soft continuum planar manipulators
}

\author{
Enrico Franco
}

\begin{abstract}
This letter investigates the model-based control of a class of soft continuum manipulators with hydraulic actuation that bend on a plane due to pressurization of one or more internal chambers. A port-Hamiltonian formulation is employed to describe the system dynamics, which includes the pressure dynamics of the hydraulic fluid. A new nonlinear control law is constructed with an energy-shaping approach, and it is combined with an adaptive observer to compensate the effect of unknown external forces. Stability conditions are investigated with a Lyapunov approach, and the effect of the tuning parameters and of key model parameters is discussed. The effectiveness of the controller is demonstrated with numerical simulations.
\end{abstract}

Index Terms-Emerging control applications, Flexible structures, Adaptive control, Stability of nonlinear systems.

\section{INTRODUCTION}

$\mathbf{S}$ OFT continuum manipulators are a class of systems characterized by high compliance and light weight, thus they are deemed ideally suited for applications that involve close contacts with delicate objects or with human operators [1]. The inherent compliance of soft manipulators however results in a large quantity of degrees of freedom (DOFs) which, together with the limited number of actuators and sensors, makes the control of these systems particularly challenging [2]. Among the various actuation principles employed for soft continuum manipulators, pneumatics and hydraulics have been particularly popular due to their high power density, ease of miniaturization, and affordability. In particular, pneumatic actuation provides fast response and it is suitable to force control, while hydraulic actuation has the ability to transmit larger forces, thus it can be beneficial for miniaturized manipulators. Other actuation strategies, such as cables [3], require more complex designs which could hamper miniaturization.

If the manipulators operate in quasi-static conditions, they can be controlled by relying solely on reduced-order kinematic models, such as the constant curvature (CC) or the piece-wise constant curvature (PCC) models. This approach however, is only valid in the absence of external forces. Instead, a reducedorder dynamical model of the system is typically necessary in case of fast movements [4]. A variety of classical modelbased controllers have been implemented for soft continuum

This research was supported by the Engineering and Physical Sciences Research Council (grant EP/W004224/1 and grant EP/R511547/1). Enrico Franco is with the Mechanical Engineering Department, Imperial College London, UK, Exhibition Road, SW7 2AZ. Contacts: e.franco11@imperial.ac.uk. manipulators, including model predictive control (MPC), sliding mode control (SMC) [5], [6], and feedback linearization [4]. However, research has shown that classical high-gain controllers can increase the closed-loop stiffness of the system, potentially reducing the benefits of soft manipulators [7]. As a result, recent approaches have been combining feedback actions that employ low gains with feed-forward actions [8] or with adaptive algorithms [9]. Nevertheless, most of the controllers in the literature do not account for the pressure dynamics of the fluid and treat the system as fully actuated. In our earlier work we proposed an energy-shaping control law for underactuated soft manipulators that included either an adaptive algorithm [10], [11] or an integral action [12] to compensate external disturbances. The former results were extended to account for the pressure dynamics of the pneumatic actuation in [13], but hydraulic actuation was not investigated.

This letter investigates the regulation problem for a class of soft continuum planar manipulators with hydraulic actuation. The complete system dynamics, including the pressure dynamics of the fluid, is described with a port-Hamiltonian formulation by introducing standard simplifying assumptions. A new control law is then constructed for the proposed dynamical model by employing the Interconnection and damping assignment Passivity based control methodology (IDA-PBC) [14]. An adaptive observer is designed with the Immersion and Invariance (I\&I) method [15], and it is included in the control law to compensate the effect of disturbances. This approach is well suited to underactuated systems, it does not rely on high gains, and it provides a physical interpretation of the control action in terms of mechanical energy. To the best of the author's knowledge, this is the first attempt to design an energy-shaping controller for soft continuum manipulators with hydraulic actuation that explicitly accounts for the pressure dynamics. The effectiveness of the controller is demonstrated with numerical simulations. The effects of the tuning parameters and of the fluid volume are also discussed.

The rest of the paper is organized as follows: the dynamical model of the system is introduced in Section II; the controller design is outlined in Section III; the results of numerical simulations are presented in Section IV; conclusions and future work are discussed in Section V.

\section{DYNAMICAL MODEL}

The class of systems considered in this work includes soft continuum manipulators, such as [16], which have one or more internal chambers and an inextensible central axis to prevent 


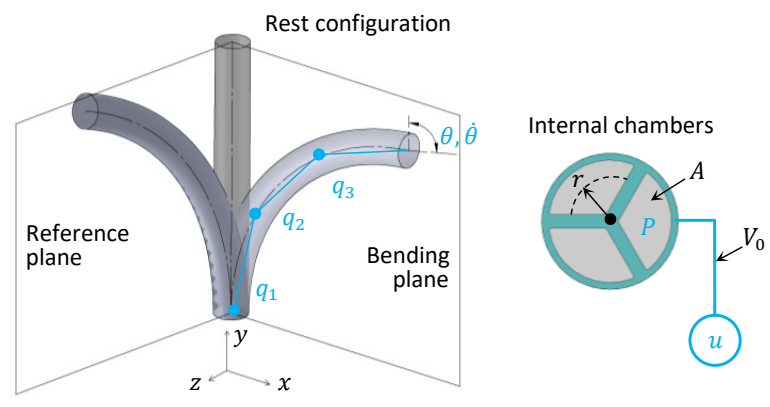

Fig. 1. Schematic of a soft manipulator and of its rigid-link model with $n=3$; section view of the internal chambers with key model parameters

elongation. The control input corresponds to the volumetric flow rate of fluid entering through a pipe in the actuated chamber, which results in the manipulator bending on a plane. In practice, the flow rate could be provided by a syringe pump actuated by a stepper motor [17]. At equilibrium and in the absence of external forces, the tip rotation $\theta$ depends on the pressure of the fluid $P$, relative to atmosphere, according to

$$
\theta=P / k^{\prime},
$$

where $k^{\prime}$ is the structural stiffness of the manipulator [18].

The dynamics of the soft continuum manipulator on the bending plane can be approximated with a rigid-link model that has $n$ virtual elastic pin joints of stiffness $k$ in series [19] (see Figure 1). Indicating with $q_{i}$ the angle of joint $i$ relative to link $i-1$ yields $\theta=\sum_{i=1}^{n} q_{i}$ and $k=n k^{\prime}$. This approach is based on the pseudo-rigid-body model which approximates the force/deflection relationship of a flexible mechanism by introducing virtual elastic joints [20]. Alternative modelling approaches include the Cosserat rod and the Euler-Bernoulli beam. The rigid-link model has $n$ DOFs and one control input, thus it is underactuated, and its dynamics can be expressed in port-Hamiltonian form as a function of its mechanical energy $H_{0}(q, \dot{q})=\frac{1}{2} \dot{q}^{T} M \dot{q}+\Omega$, where $M(q)=M^{T}(q)>0$ is the inertia matrix and $\Omega(q)$ is the potential energy [10]. To simplify the notation, the arguments of $H_{0}, M$, and $\Omega$ are omitted in the rest of the paper. The following assumptions are introduced for control purposes.

Assumption 1: The fluid is isentropic and inviscid, and its bulk modulus $\Gamma_{0}$ is constant and known. In addition, the pressure $P$ of the fluid, its density $\rho$ and speed $v$ are uniform. Finally, the system is horizontal, thus the height $z$ of the fluid with respect to a reference is constant and uniform.

Assumption 2: The tip rotation $\theta$ and its time derivative $\dot{\theta}$ are known at any instant and are bounded. In addition, $q_{i} q_{j}>$ $0, \forall i, j$, thus all sections of the soft manipulator bend in the same direction, however $q_{i}$ are not measurable. The pressure $P$ is known at any instant and bounded, such that $P \ll \Gamma_{0}$.

Assuming constant bulk modulus is realistic for water at low pressures (e.g. $P \leq 10$ bar) and at ambient temperature [21]. Assuming uniform pressure, density, and speed of the fluid is a reasonable approximation in case of laminar flow conditions. Neglecting the effect of viscosity is appropriate at low speeds (see Section IV). Finally, assuming that the system lies in the horizontal plane simplifies the potential energy, which becomes $\Omega=\frac{k}{2} \sum_{i=1}^{n} q_{i}^{2}$ hence it only includes elastic terms. The angle $\theta$ can be measured with an electromagnetic tracking system and its time derivative $\dot{\theta}$ can be computed by discrete differentiation (see [11]), while the pressure $P$ can be measured with a sensor (see [13]). Finally, Assumption 2 is less stringent than CC, which implies $q_{i}=q_{j} \forall i, j$.

For inextensible soft manipulators, the volume variation of the chamber can be approximated as $V_{c}=\operatorname{Ar} \sum_{i=1}^{n}\left|q_{i}\right|$, where $A$ is the cross-section area of the internal chamber and $r$ is the distance from the centroid of the chamber to the center of the section [13]. If all sections of the manipulator bend in the same direction, the volume of the fluid can be expressed as $V=V_{0}+\operatorname{Ar} \theta$, where $V_{0}$ includes the volume of the supply pipes. It follows from Assumption 1 and 2 that the speed of the fluid flow can be approximated as $v=r \dot{\theta}$. Thus the mechanical energy of the isentropic fluid can be computed as the sum of the internal energy [22] and the kinetic energy of the fluid

$$
\Phi=\left(-P+\Gamma_{0}\left(e^{P / \Gamma_{0}}-1\right)+\frac{1}{2} \rho r^{2} \dot{\theta}^{2}\right)\left(V_{0}+A r \theta\right) .
$$

The pressure dynamics is given by

$$
\dot{P}=\Gamma_{0} \frac{u-A r \dot{\theta}}{V_{0}+A r \theta},
$$

where the volumetric flow rate $u$ corresponds to the control input [23]. Including (2) in the mechanical energy of the system yields $H=\frac{1}{2} \dot{q}^{T} M \dot{q}+\Omega+\Phi$. Combining all kinetic energy terms together, the inertia matrix can be expressed as $\widehat{M}=M+\rho r^{2} V G G^{T}$, where $G$ is a column vector with all elements equal to 1 , while $M$ is computed as in [10]. The complete system dynamics in port-Hamiltonian form is thus

$$
\left[\begin{array}{c}
\dot{q} \\
\dot{p} \\
\dot{P}
\end{array}\right]=\left[\begin{array}{ccc}
0 & I & 0 \\
-I & -D & \frac{\Gamma_{0} A r}{V} \\
0 & -\frac{\Gamma_{0} A r}{V} & 0
\end{array}\right]\left[\begin{array}{c}
\nabla_{q} H \\
\nabla_{p} H \\
\nabla_{P} H
\end{array}\right]+\left[\begin{array}{c}
0 \\
-\delta \\
\frac{\Gamma_{0}}{V} u
\end{array}\right],
$$

where the system states are the position $q \in \mathbb{R}^{n}$ of the virtual joints, the momenta $p=\widehat{M} \dot{q}$, and the pressure $P$. External forces and model uncertainties are included in the term $\delta \in \mathbb{R}^{n}$ which is unknown and time-varying, while $D$ indicates the physical damping. The term $I$ indicates the identity matrix of dimensions $n \times n$, and $\nabla_{q} H$ represents the vector of partial derivatives in $q$. System (4) is further qualified by the following assumptions [11].

Assumption 3: The model parameters in (4) are accurately known. In particular, the stiffness $k$ and the physical damping $D>D_{0}+D_{1}|\dot{q}|^{2}>0$ are uniform along the length, with $D_{0}>0, D_{1}>0$ and $k>0$ known scalar constants.

Assumption 4: The disturbance $\delta$ is unknown, and it can be parameterized as $\delta=\delta_{0}+\sigma$, where $\delta_{0}$ is the constant unknown part, while $\sigma$ is the time-varying zero-mean bounded part, with $|\sigma| \leq \sigma_{0}|\dot{q}|$ for some known $\sigma_{0}>0$.

The model parameters are either known from the design, or they can be estimated experimentally (see [11] for damping identification). In practice, the disturbances $\delta$ can also include the effect of gravity, similarly to [10]. Computing $\dot{P}$ from (4) 
recovers the pressure dynamics (3), while computing $\dot{p}$ yields

$$
\dot{p}=-\nabla_{q} \Omega-\frac{1}{2} \nabla_{q}\left(p^{T} \widehat{M}^{-1} p\right)-D \dot{q}-\delta+P A r G,
$$

which shows that the pressure of the fluid acts in a uniform fashion on all $n$ virtual joints through the matrix $G$.

\section{ENERGY-SHAPING CONTROL}

\section{A. Adaptive observer}

The effect of $\delta_{0}$ on the tip rotation $\theta$, that is $\frac{1}{n} G^{T} \delta_{0}$, is estimated with the observer $\tilde{\delta}_{0}=\widehat{\delta}_{0}+\beta_{0}$ where

$$
\begin{array}{r}
\dot{\widehat{\delta}}_{0}=-\alpha\left(\frac{k}{n} \theta+\frac{1}{2} \rho A r^{3} \dot{\theta}^{2}+\frac{D+\sigma_{0}}{n} \dot{\theta}+\tilde{\delta}_{0}-P A r\right), \\
\beta_{0}=-\alpha r^{2} \rho\left(V_{0}+A r \theta\right) \dot{\theta},
\end{array}
$$

with $\alpha$ a tuning parameter, and $\widehat{\delta}_{0}$ is the observer state.

Proposition 1: Consider system (4) with Assumptions 1 to 4 and with the adaptive law (6). Define the estimation error as $\zeta=\widehat{\delta}_{0}-\frac{\alpha}{n} G^{T} p-\frac{1}{n} G^{T} \delta_{0}$, where $\widehat{\delta}_{0}$ is computed from (6). Then $\zeta$ is ultimately bounded for all $\alpha>1 / 4$.

Proof: Computing the time derivative of $\zeta$ and substituting $\dot{p}$ from (5) and $\delta_{0}$ from $\zeta$, where $\delta=\delta_{0}+\sigma$, gives

$$
\begin{array}{r}
\dot{\zeta}=\dot{\widehat{\delta}}_{0}-\frac{\alpha}{n} G^{T} \dot{p} \\
=\dot{\widehat{\delta}}_{0}+\alpha\left(\frac{1}{n} G^{T} \nabla_{q} \Omega+\frac{1}{2 n} G^{T} \nabla_{q}\left(p^{T} \widehat{M}^{-1} p\right)-P A r\right) \\
+\alpha\left(\frac{1}{n} D \dot{\theta}+\frac{1}{n} G^{T} \sigma+\left(\widehat{\delta}_{0}-\frac{\alpha}{n} G^{T} p-\zeta\right)\right) .
\end{array}
$$

Substituting (6) into (7), refactoring terms, and noting that $\frac{\alpha}{n} G^{T} p=\frac{\alpha}{n} G^{T} M \dot{q}-\beta_{0}$ yields

$\dot{\zeta}=\frac{\alpha}{n}\left(\frac{1}{2} G^{T} \nabla_{q}\left(\dot{q}^{T} M \dot{q}\right)-\alpha G^{T} M \dot{q}-n \zeta+G^{T} \sigma-\sigma_{0} \dot{\theta}\right)$.

The kinetic terms in (8) only depend on the inertia of the rigidlink model, but not on that of the fluid. Also, $\max \{M\}<$ $m_{T} l_{T}^{2}$, with $m_{T}$ the total mass of the links, $l_{T}$ their total length, and $\max \{M\}$ the largest element of $M$ [10]. Thus the inequalities $|M \dot{q}| \leq c_{1} m_{T} l_{T}^{2}|\dot{q}|$ and $\left|\nabla_{q}\left(\dot{q}^{T} M \dot{q}\right)\right| \leq$ $c_{2} m_{T} l_{T}^{2}|\dot{q}|^{2}$ hold for some $0<c_{1}, c_{2}<1$. Defining the Lyapunov function candidate $\Upsilon=\frac{1}{2} \zeta^{2}$, computing its time derivative, substituting (8) and the former inequalities yields

$\dot{\Upsilon} \leq-\alpha \zeta^{2}+\frac{\alpha}{n}|\zeta|\left(\frac{n}{2} c_{2} m_{T} l_{T}^{2}|\dot{q}|^{2}+\left(\alpha c_{1} m_{T} l_{T}^{2}+\sigma_{0}\right)|\dot{\theta}|\right)$,

which can be rewritten as $\dot{\Upsilon} \leq-\alpha \zeta^{2}+\alpha|\zeta| \epsilon$, where $\epsilon$ depends on $|\dot{q}|$. Introducing the Young's inequality $\alpha|\zeta| \epsilon \leq \zeta^{2} / 4+\alpha^{2} \epsilon^{2}$ and substituting it in (9) yields $\dot{\Upsilon} \leq-\left(\alpha-\frac{1}{4}\right) \zeta^{2}+\alpha^{2} \epsilon^{2}$. It follows from Assumption 2 that $|\dot{q}|$ and $\epsilon$ are bounded, thus $\zeta$ is bounded for all $\alpha>1 / 4$ concluding the proof

Remark 1: Differently from our prior works [10], [13], the adaptive observer (6) accounts for the inertia of the fluid. Conversely, the inertia of the rigid-link model, which depends on the virtual positions $q_{i}$, is not included in (6), and the time-varying disturbances $\sigma$ are not canceled exactly. As a result, Proposition 1 does not ensure convergence of $\zeta$ to zero, however (6) is implementable since it only depends on $\theta, \dot{\theta}$ and $P$, which are assumed measurable (see Assumption 2), but not on $\zeta$ or $\delta_{0}$. Finally, the condition $\alpha>1 / 4$ is sufficient since it results from Young's inequalities which are conservative.

\section{B. Controller design}

The control law is designed to achieve the regulation goal $\theta=\theta^{*}$ following a similar procedure to [13] such that the closed-loop dynamics in port-Hamiltonian form becomes

$$
\left[\begin{array}{c}
\dot{q} \\
\dot{p} \\
\dot{P}
\end{array}\right]=\left[\begin{array}{ccc}
0 & S_{12} & S_{13} \\
-S_{12}^{T} & -S_{22} & S_{23} \\
-S_{13}^{T} & -S_{23}^{T} & -S_{33}
\end{array}\right]\left[\begin{array}{c}
\nabla_{q} H_{d} \\
\nabla_{p} H_{d} \\
\nabla_{P} H_{d}
\end{array}\right]-\left[\begin{array}{c}
0 \\
\sigma \\
0
\end{array}\right],
$$

where $H_{d}=\Omega_{d}+\frac{1}{2} p^{T} \widehat{M}_{d}^{-1} p+\varsigma^{2} / 2$ is a positive definite and radially unbounded storage function, and $\varsigma$ is defined as

$$
\varsigma=-\frac{k \theta}{n}-k_{p}\left(\theta^{*}-\theta\right)-\tilde{\delta}_{0}+P A r
$$

with $k_{p}$ a tuning parameter, $\tilde{\delta}_{0}$ computed from (6), and $\theta^{*}$ the prescribed rotation. The terms $S_{\mathrm{ij}}$ are defined so that the openloop dynamics (4) matches the closed-loop dynamics (10).

Step 1: Equating the first rows of (4) and of (10) gives

$$
\widehat{M}^{-1} p=S_{12} \widehat{M}_{d}^{-1} p+S_{13} A r \varsigma .
$$

Setting $S_{12}=k_{m} I, S_{13}=0$, and $\widehat{M}_{d}=k_{m} \widehat{M}$, with $k_{m}>0$ a tuning parameter that scales the kinetic energy, verifies (12).

Step 2: Equating the second rows of (4) and of (10) yields

$$
\begin{array}{r}
-\nabla_{q} \Omega-\frac{1}{2} \nabla_{q}\left(p^{T} \widehat{M}^{-1} p\right)-D \widehat{M}^{-1} p-\delta+P A r G= \\
-S_{12}^{T}\left(\nabla_{q} \Omega_{d}+\frac{1}{2} \nabla_{q}\left(p^{T} \widehat{M}_{d}^{-1} p\right)+\varsigma\left(-\frac{k}{n}+k_{p}\right) G\right) \\
-S_{22} \widehat{M}_{d}^{-1} p+S_{23} A r G \varsigma-\sigma .
\end{array}
$$

Substituting $S_{12}=k_{m} I, \widehat{M}_{d}=k_{m} \widehat{M}$, and $S_{22}=k_{m} D$ the kinetic energy terms and the damping terms vanish from (13). Pre-multiplying both sides of (13) with the matrix $G^{\perp}$, where $G^{\perp} G=0$ and rank $\left\{G^{\perp}\right\}=n-1$, yields the following partial differential equations (PDEs)

$$
\begin{gathered}
G^{\perp}\left(\nabla_{q} \Omega-k_{m} \nabla_{q}\left(\Omega_{d}-\Omega_{0}\right)\right)=0, \\
G^{\perp}\left(\delta_{0}-k_{m} \nabla_{q} \Omega_{0}\right)=0,
\end{gathered}
$$

where $\Omega_{0}=\Lambda^{T}\left(q-\theta^{*} / n\right)$, and $\Lambda$ can be interpreted as a vector of closed-loop non-conservative forces [24]. Solving (14) and (15) while enforcing the minimizer conditions $\nabla_{q} \Omega_{d}\left(\theta^{*}\right)=0$ and $\nabla_{q}^{2} \Omega_{d}\left(\theta^{*}\right)>0$ in the presence of a constant $\delta_{0}$ yields

$$
\Omega_{d}=\frac{k}{2 k_{m}}\left(\sum_{i=1}^{n} q_{i}^{2}+\frac{k_{p}}{k}\left(\theta-\theta^{*}\right)^{2}-\frac{\theta^{2}}{n}\right)+\Lambda^{T}\left(q-\frac{\theta^{*}}{n}\right),
$$

where $k_{p}>0$ was defined in (11) and $\Lambda$ has $n$ components

$$
\Lambda_{i}=\frac{1}{n k_{m}}\left((n-1) \delta_{0_{i}}-\sum_{j=1}^{n} \delta_{0_{j \neq i}}\right) \text {. }
$$


Substituting (16) and (17) in (13) and multiplying it by the pseudo-inverse $G^{\dagger}=\left(G^{T} G\right)^{-1} G^{T}=\frac{1}{n} G^{T}$ yields

$$
S_{23}=\frac{1+k_{m}\left(-\frac{k}{n}+k_{p}\right)}{A r} .
$$

Step 3: Equating the third rows of (4) and of (10) yields

$$
\Gamma_{0} \frac{u-A r \dot{\theta}}{V_{0}+A r \theta}=-\frac{S_{23}}{k_{m}} \dot{\theta}-S_{33} A r \varsigma .
$$

Computing the control input $u$ from (19), substituting (18), and defining $S_{33}=\frac{k_{i}}{A r}>0$ yields finally the control law

$$
\begin{gathered}
u=A r \dot{\theta}-\frac{\left(V_{0}+A r \theta\right)}{\Gamma_{0}} \frac{\left(1+k_{m}\left(-\frac{k}{n}+k_{p}\right)\right)}{k_{m} A r} \dot{\theta} \\
+\frac{\left(V_{0}+A r \theta\right)}{\Gamma_{0}} k_{i}\left(\frac{k}{n} \theta+k_{p}\left(\theta^{*}-\theta\right)-P A r+\tilde{\delta}_{0}\right),
\end{gathered}
$$

where the tuning parameters are $k_{m}, k_{p}, k_{i}$, and $\alpha$ in (6). Note that substituting $k=n k^{\prime}$ in (6) and in (20), where $k^{\prime}$ can be estimated experimentally with (1), the control law does not depend on $n$. The same holds for the damping parameters.

Remark 2: The key differences between (20) and the corresponding controller for pneumatic actuation are the pressure dynamics in (19) and the bulk modulus $\Gamma_{0}$. For an incompressible fluid (e.g. $\Gamma_{0} \rightarrow \infty$ ), the control law (20) reduces to $u=A r \dot{\theta}$, thus it is proportional to the angular velocity of the tip. In case $\Gamma_{0}$ is finite but the volume of the pipe is large (i.e. $V_{0} \gg A r|\theta|$ ), the control law (20) can be simplified as

$$
\begin{array}{r}
u=A r \dot{\theta}-\frac{V_{0}}{\Gamma_{0}} \frac{\left(1+k_{m}\left(-\frac{k}{n}+k_{p}\right)\right)}{k_{m} A r} \dot{\theta} \\
+\frac{V_{0}}{\Gamma_{0}} k_{i}\left(\frac{k}{n} \theta+k_{p}\left(\theta^{*}-\theta\right)-P A r+\tilde{\delta}_{0}\right),
\end{array}
$$

which can be rewritten in a more compact form as

$$
u=-K_{v} \dot{\theta}+K_{i}\left(\frac{k}{n} \theta+k_{p}\left(\theta^{*}-\theta\right)-P A r+\tilde{\delta}_{0}\right) .
$$

Note that (21) is very similar to the case of controlled pressure [10], where pressure dynamics was neglected. The tuning parameters in (21) are $K_{v}$, which multiplies the velocity $\dot{\theta}$, $K_{i}$ and $k_{p}$ which multiply the error $\left(\theta^{*}-\theta\right)$, and $\alpha$ from (6). In addition, (21) depends on the model parameters $k, A, r, n$. Controller (20) also has four tuning parameters and depends on the same model parameters, and also on $\Gamma_{0}$. A comparison between (20) and (21) is provided in Section IV.

\section{Stability analysis}

Proposition 2: Consider system (4) with Assumptions 1 to 4 in closed-loop with the control law (20), where the adaptive estimate $\tilde{\delta}_{0}$ is computed with (6). Define the parameters $k_{i}, k_{m}, \alpha$ such that the symmetric matrix

$$
\Theta=\left[\begin{array}{cccc}
\frac{I D_{0}^{\prime}}{k_{m}} & 0 & 0 & \star \\
0 & \frac{D_{1}}{k_{m}} & 0 & \star \\
0 & 0 & k_{i} A r & 0 \\
-\left(\frac{1}{2 k_{m}}+\frac{\Sigma_{0}}{2}\right) & -\frac{\alpha c_{2} m_{T} l_{T}^{2}}{4} & 0 & \alpha
\end{array}\right],
$$

is positive definite for some values $0<c_{1}, c_{2}<1$, where $D_{0}^{\prime}=\left(D_{0}-\alpha c_{1} m_{T} l_{T}^{2}-\sigma_{0}\right)$ and $\Sigma_{0}=\alpha^{2} c_{1} m_{T} l_{T}^{2}+\alpha \sigma_{0}$.
Then the equilibrium point $(\theta, \dot{\theta})=\left(\theta^{*}, 0\right)$ is stable and $\theta$ converges to $\theta^{*}$ asymptotically. Additionally, $\theta^{*}=\operatorname{argmin}\left\{\Omega_{d}\right\}$ for all $k_{p}, k_{m}>0$.

Proof: Substituting (20) into (4) results in the closed-loop dynamics

$$
\left[\begin{array}{c}
\dot{q} \\
\dot{p} \\
\dot{P}
\end{array}\right]=\left[\begin{array}{ccc}
0 & +k_{m} I & 0 \\
-k_{m} I & -k_{m} D & \frac{1+k_{m} \lambda}{A r_{i}} \\
0 & -\frac{1+k_{m} \lambda}{A r} & -\frac{k_{i}}{A r}
\end{array}\right]\left[\begin{array}{c}
\nabla_{q} H_{d} \\
\nabla_{p} H_{d} \\
\nabla_{P} H_{d}
\end{array}\right]+\left[\begin{array}{c}
0 \\
\sigma^{\prime} \\
0
\end{array}\right],
$$

where $\lambda=\left(-\frac{k}{n}+k_{p}\right)$ and $\sigma^{\prime}=G \zeta+\alpha M \dot{q}-\sigma$.

Defining the Lyapunov function $\Psi=H_{d}+\Upsilon$ and computing its time derivative along the trajectories of (23) yields

$$
\begin{array}{r}
\dot{\Psi} \leq-\nabla_{p} H_{d}{ }^{T} k_{m} D \nabla_{p} H_{d}-\alpha \zeta^{2} \\
-\frac{k_{i}}{A r} \nabla_{P} H_{d}{ }^{2}+\nabla_{p} H_{d}{ }^{T}(G \zeta+\alpha M \dot{q}-\sigma) \\
+\frac{\alpha}{n}|\zeta|\left(\frac{1}{2} G^{T} \nabla_{q}\left|\dot{q}^{T} M \dot{q}\right|+\alpha\left|G^{T} M \dot{q}\right|+\sigma_{0}|\dot{\theta}|\right) .
\end{array}
$$

Introducing the same inequalities as in (9) yields

$$
\begin{aligned}
\dot{\Psi} \leq- & \frac{1}{k_{m}}\left(D_{0}+D_{1}|\dot{q}|^{2}\right) \dot{q}^{2}-\alpha \zeta^{2}-k_{i} \varsigma^{2} A r \\
& +\frac{1}{k_{m}}|\dot{q}||\zeta|+\frac{1}{k_{m}}|\dot{q}|^{2}\left(\alpha c_{1} m_{T} l_{T}^{2}+\sigma_{0}\right) \\
+\alpha|\zeta| & \left(\frac{1}{2} c_{2} m_{T} l_{T}^{2}|\dot{q}|^{2}+\left(\alpha c_{1} m_{T} l_{T}^{2}+\sigma_{0}\right)|\dot{q}|\right) .
\end{aligned}
$$

Refactoring common terms in (25) yields

$$
\dot{\Psi} \leq-\bar{x}^{T} \Theta \bar{x},
$$

where $\bar{x}^{T}=\left[\begin{array}{llll}\dot{q}^{T} & \left(\dot{q}^{2}\right)^{T} & \varsigma & \zeta\end{array}\right]$ and $\Theta$ is given in (22). Thus $\dot{\Psi} \leq 0$, hence $\bar{x}$ is bounded and converges to zero asymptotically, and the equilibrium point $\bar{x}=0$ is stable. Computing $\dot{p}$ from (23) at $\bar{x}=0$ yields $\nabla_{q} \Omega_{d}=0$, which implies that $H_{d}$ has an extremum at $\bar{x}=0$. Computing $G^{T} \nabla_{q} \Omega_{d}$ from (16) while noting that $G^{T} \Lambda=0$ from (17), and computing the minimizer condition $\nabla_{q}^{2} \Omega_{d}\left(\theta^{*}\right)>0$ yields

$$
\begin{array}{r}
G^{T} \nabla_{q} \Omega_{d}=k_{p} n\left(\theta-\theta^{*}\right) / k_{m}=0, \\
\operatorname{det}\left(\nabla_{q}^{2} \Omega_{d}\right)=n k_{p} k^{n-1} / k_{m}^{n}>0,
\end{array}
$$

which hold true at $\theta=\theta^{*}$ for all $k_{p}, k_{m}>0$. Thus $\theta$ converges to $\theta^{*}$ asymptotically concluding the proof

Remark 3: Note that (22) does not depend on either $V_{0}$ or $\Gamma_{0}$, and in case $m_{T} l_{T}^{2} \ll 1$ it can be simplified as

$$
\Theta=\left[\begin{array}{cccc}
\frac{I\left(D_{0}-\sigma_{0}\right)}{k_{m}} & 0 & 0 & -\frac{\alpha \sigma_{0}}{2}-\frac{1}{2 k_{m}} \\
0 & \frac{D_{1}}{k_{m}} & 0 & 0 \\
0 & 0 & k_{i} A r & 0 \\
-\frac{\alpha \sigma_{0}}{2}-\frac{1}{2 k_{m}} & 0 & 0 & \alpha
\end{array}\right] .
$$

A necessary condition to ensure $\Theta>0$ in (28) is thus

$$
D_{0}>\sigma_{0}+\frac{\left(\alpha \sigma_{0} k_{m}+1\right)^{2}}{4 k_{m} \alpha},
$$

hence $D_{0}$ should be sufficiently large to dominate the effect of time-varying disturbances. In addition, increasing the product $\alpha k_{m}$ reduces the contribution of $\sigma_{0}$ as long as $\alpha k_{m} \sigma_{0}<1$, 
while it has the opposite effect if $\alpha k_{m} \sigma_{0}>1$. Note finally that the effect on stability of the time-varying disturbances $\sigma$ is similar to that of an uncertain damping, since the bound $\sigma_{0}$ appears alongside $D_{0}$ in (22) and in (28).

\section{SIMULATIONS}

Simulations have been conducted in Matlab for system (4) employing the model parameters $k=2, A=56.5, r=0.003$, and $D_{0}=0.05, D_{1}=0.01$ for illustrative purposes. The model consists of $n=3$ virtual links of equal length with mass concentrated at their midpoint, thus $k^{\prime}=2 / 3$. The total mass and the total length of the links are $m_{T}=1.5$ and $l_{T}=0.015$. The links at rest are collinear (i.e. $q=0$ ) and an unmodeled external force $f_{0}$ parallel to the neutral axis at rest acts on the tip of the distal link resulting in an unknown bending moment opposite to positive $\theta$. The remaining parameters are the pipe volume $V_{0}=10 A l_{T}$, the bulk modulus $\Gamma_{0}=20000$, and the density $\rho=1$ for water. The tuning parameters for controller (20) have been set as $k_{p}=0.5, k_{m}=2.5, k_{i}=30, \alpha=5$. These values ensure $\Theta>0$ in (22) and (29) for all $\sigma_{0} \leq D_{0} / 4$. The initial conditions are $q=0, p=0, P=0, \widehat{\delta}_{0}=0$.

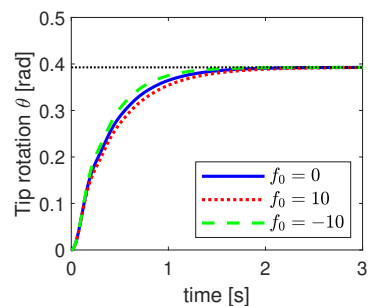

(a)

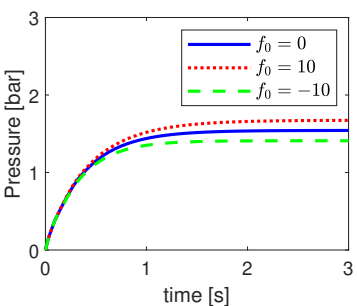

(c)

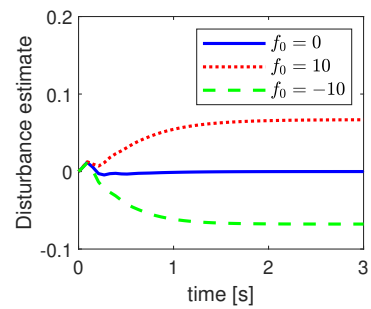

(e)

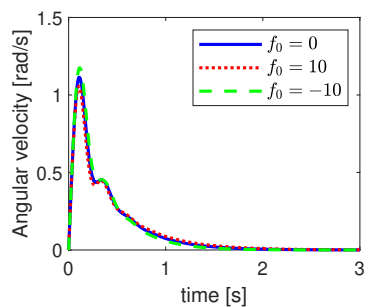

(b)

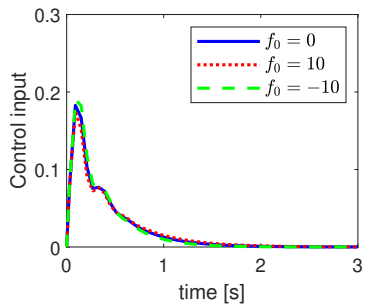

(d)

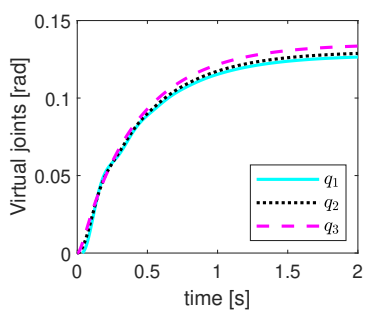

(f)
Fig. 2. Simulation results for system (4) with controller (20) and different values of $f_{0}$ : (a) tip rotation $\boldsymbol{\theta}$; (b) angular velocity $\dot{\boldsymbol{\theta}}$; (c) gauge pressure $\boldsymbol{P}$; (d) control input; (e) disturbance estimate $\tilde{\delta_{0}}$; (f) virtual joints $\boldsymbol{q}_{\boldsymbol{i}}$ for $f_{0}=10$.

The simulation results in Figure 2 show the time histories of the tip rotation $\theta$ for system (4) with different values of the unmodeled tip force $f_{0}$. This condition is also representative of an unknown mass comparable to $m_{T}$ attached to the tip

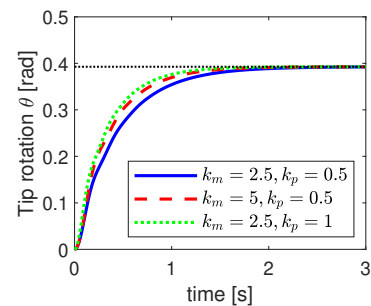

(a)

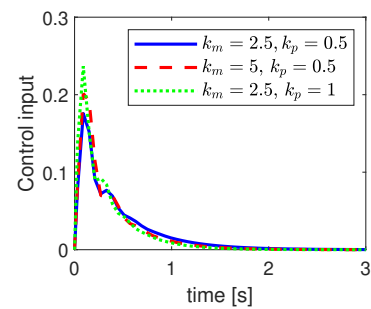

(c)

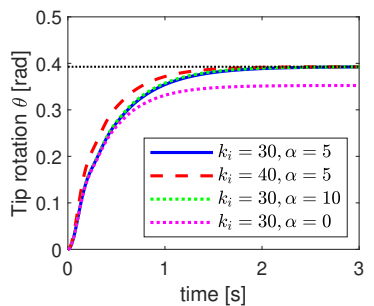

(b)

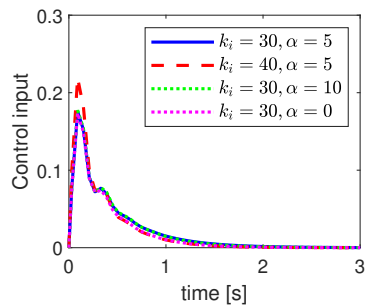

(d)
Fig. 3. Simulation results for controller (20) with $\boldsymbol{f}_{\mathbf{0}}=\mathbf{1 0}$ and different values of the tuning parameters: (a) time history of the tip rotation $\boldsymbol{\theta}$ for different values of $\boldsymbol{k}_{\boldsymbol{p}}$ and $\boldsymbol{k}_{\boldsymbol{m}}$; (c) control input; (b) tip rotation $\boldsymbol{\theta}$ for different values of $\boldsymbol{k}_{\boldsymbol{i}}$ and $\boldsymbol{\alpha}$; (d) control input. The tuning parameters are $k_{p}=0.5 ; k_{m}=2.5 ; k_{i}=30 ; \alpha=5$ unless otherwise stated in the legend.

of the manipulator if the latter moves on a vertical plane (see [10], [12]). The controller (20) achieves the regulation goal with a consistent transient using the same tuning parameters. The disturbance estimate corresponds to the bending moment produced by $f_{0}$, and the pressure $P$ varies accordingly. Note that the joint angles $q_{i}$ have the same sign but not the same values in case $f_{0} \neq 0$. While there is a close similarity between control input and velocity, the regulation goal fails if $u=A r \dot{\theta}$ (i.e. $\Gamma_{0} \rightarrow \infty$ ), since $u=0$ starting from rest conditions.

The effect of the tuning parameters in controller (20) is shown in Figure 3. A larger $k_{m}$, a larger $k_{p}$, and a larger $k_{i}$ result in a faster transient but also in higher control action. The effect of $\alpha$ is less pronounced, provided that the stability conditions are satisfied. In particular, setting $\alpha=0$ results in steady-state errors since the disturbances are not compensated. In addition, exceedingly high values of $\alpha$ and $k_{m}$ might lead to vibrations in case of low $D_{0}$ or of large $\sigma_{0}$ (see Remark 3).

The system response with different pipe volumes $V_{0}$ is shown in Figure 4. In particular, the controller (21) with $K_{v}=S_{23} V_{0} /\left(A r k_{m} \Gamma_{0}\right)-A r, K_{i}=k_{i} V_{0} / \Gamma_{0}$ yields similar performance to (20) provided that $V_{0} \gg A r\left|\theta^{*}\right|$ (see Remark 2). Conversely, the controller (21) results in a slower response for smaller $V_{0}$. Finally, the speed of the fluid remains below $10^{-2} \mathrm{~m} / \mathrm{s}$, thus the effects of the dynamic viscosity would be in the range $10^{-5} \mathrm{~Pa}$, confirming that Assumption 1 is realistic.

\section{CONCLUSIONS}

In this letter a new energy-shaping controller for planar soft continuum manipulators with hydraulic actuation has been presented. The controller accounts for the pressure dynamics of the fluid, and an adaptive observer is employed to compensate the effect of disturbances. The simulation results indicate that 


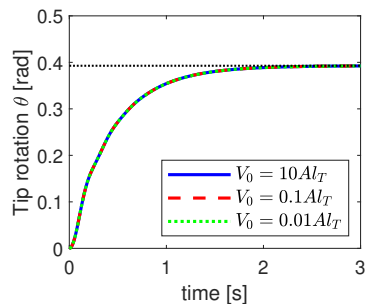

(a)

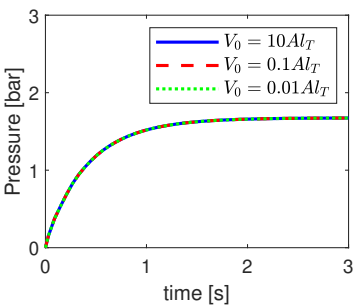

(c)

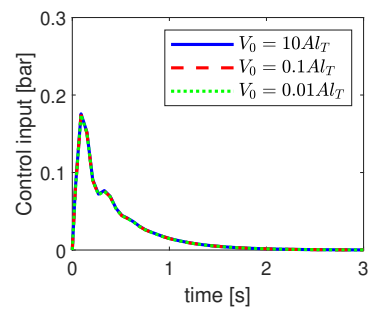

(e)

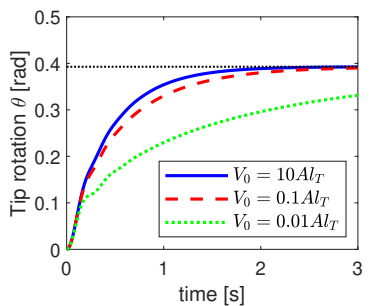

(b)

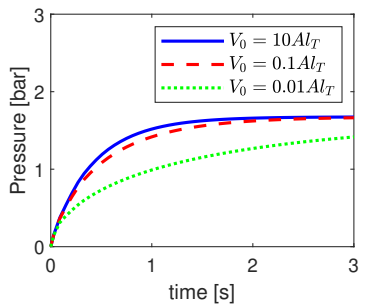

(d)

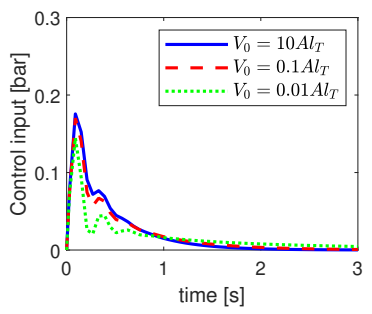

(f)
Fig. 4. Simulation results for system (4) with different values of $\boldsymbol{V}_{\mathbf{0}}$ : (a) tip rotation $\boldsymbol{\theta}$ with controller (20); (c) gauge pressure $\boldsymbol{P}$; (e) control input; (b) tip rotation $\boldsymbol{\theta}$ with controller (21); (d) gauge pressure $\boldsymbol{P}$; (f) control input.

the controller correctly achieves the regulation goal with a smooth control action and a consistent transient in the presence of unmodeled external forces. In comparison, a simplified control law shows similar performance for a large pipe volume, but it results in a slower response otherwise. Future work will aim to relax the initial assumptions, to model the dynamics of the pump, and to consider different types of disturbances, such as gravity, in an explicit fashion. Alternatives to the rigidlink model, such as the Cosserat rod and the Euler-Bernoulli beam, will also be investigated. Finally, the controller shall be evaluated experimentally and shall be compared to alternative approaches such as control-by-interconnection.

\section{REFERENCES}

[1] M. Runciman, A. Darzi, and G. P. Mylonas, "Soft Robotics in Minimally Invasive Surgery," Soft Robotics, vol. 6, pp. 423-443, mar 2019.

[2] T. G. Thuruthel, Y. Ansari, E. Falotico, and C. Laschi, "Control Strategies for Soft Robotic Manipulators: A Survey," Soft Robotics, vol. 5, pp. 149-163, jan 2018.

[3] M. Runciman, J. Avery, M. Zhao, A. Darzi, and G. P. Mylonas, "Deployable, Variable Stiffness, Cable Driven Robot for Minimally Invasive Surgery," Frontiers in Robotics and AI, vol. 6, p. 141, jan 2020.

[4] C. Wang, C. G. Frazelle, J. R. Wagner, and I. Walker, "Dynamic Control of Multi-Section Three-Dimensional Continuum Manipulators Based on Virtual Discrete-Jointed Robot Models," IEEE/ASME Transactions on Mechatronics, pp. 1-11, jun 2020.
[5] C. M. Best, L. Rupert, and M. D. Killpack, "Comparing model-based control methods for simultaneous stiffness and position control of inflatable soft robots," The International Journal of Robotics Research, vol. 40, pp. 470-493, may 2020

[6] G. Cao, Y. Liu, Y. Jiang, F. Zhang, G. Bian, and D. H. Owens, "Observerbased continuous adaptive sliding mode control for soft actuators," Nonlinear Dynamics, pp. 1-16, jun 2021.

[7] C. Della Santina, M. Bianchi, G. Grioli, F. Angelini, M. Catalano, M. Garabini, and A. Bicchi, "Controlling Soft Robots: Balancing Feedback and Feedforward Elements," IEEE Robotics \& Automation Magazine, vol. 24, pp. 75-83, sep 2017.

[8] C. Della Santina, R. K. Katzschmann, A. Bicchi, and D. Rus, "Modelbased dynamic feedback control of a planar soft robot: trajectory tracking and interaction with the environment," The International Journal of Robotics Research, vol. 39, pp. 490-513, jan 2020.

[9] M. Trumic, C. D. Santina, K. Jovanovic, and A. Fagiolini, "Adaptive Control of Soft Robots Based on an Enhanced 3D Augmented Rigid Robot Matching," IEEE Control Systems Letters, vol. 5, no. 6, pp. 19341939, 2021.

[10] E. Franco and A. Garriga-Casanovas, "Energy Shaping Control of Soft Continuum Manipulators with in-plane Disturbances," The International Journal of Robotics Research, vol. 40, pp. 236-255, mar 2021.

[11] E. Franco, A. Garriga-Casanovas, J. Tang, F. Rodriguez y Baena, and A. Astolfi, "Adaptive energy shaping control of a class of nonlinear soft continuum manipulators," IEEE ASME Trans Mechatron, pp. 1-11, 2021.

[12] E. Franco, A. Garriga-Casanovas, and A. Donaire, "Energy shaping control with integral action for soft continuum manipulators," Mechanism and Machine Theory, vol. 158, pp. 1-16, apr 2021.

[13] E. Franco, T. Ayatullah, A. Sugiharto, A. Garriga Casanovas, and V. Vidrdyawan, "Nonlinear energy-based control of soft continuum pneumatic manipulators," Nonlinear Dynamics, pp. 1-25, sep 2021.

[14] R. Ortega, M. Spong, F. Gomez-Estern, and G. Blankenstein, "Stabilization of a class of underactuated mechanical systems via interconnection and damping assignment," IEEE Transactions on Automatic Control, vol. 47, pp. 1218-1233, aug 2002.

[15] A. Astolfi and R. Ortega, "Immersion and invariance: A new tool for stabilization and adaptive control of nonlinear systems," IEEE Transactions on Automatic Control, vol. 48, no. 4, pp. 590-606, 2003.

[16] A. Garriga-Casanovas, I. Collison, and F. Rodriguez y Baena, "Toward a Common Framework for the Design of Soft Robotic Manipulators with Fluidic Actuation," Soft Robotics, vol. 5, pp. 622-649, aug 2018.

[17] Y. Long, Y. Zhao, S. Lu, J. Gao, P. Qu, and C. Zhang, "Dynamic modeling of syringe-pump-actuated microliquid-dispensing system by a bond-graph approach," Microsystem Technologies, vol. 26, pp. 28472855 , sep 2020.

[18] K. Suzumori, S. Iikura, and H. Tanaka, "Development of flexible microactuator and its applications to robotic mechanisms," in Proceedings. 1991 IEEE International Conference on Robotics and Automation, pp. 1622-1627, IEEE Comput. Soc. Press, 1991.

[19] I. S. Godage, R. Wirz, I. D. Walker, and R. J. Webster, "Accurate and Efficient Dynamics for Variable-Length Continuum Arms: A Center of Gravity Approach," Soft Robotics, vol. 2, pp. 96-106, sep 2015.

[20] Y.-Q. Yu, L. L. Howell, C. Lusk, Y. Yue, and M.-G. He, "Dynamic Modeling of Compliant Mechanisms Based on the Pseudo-Rigid-Body Model," Journal of Mechanical Design, vol. 127, p. 760, jul 2005.

[21] A. T. Hayward, "How to measure the isothermal compressibility of liquids accurately," Journal of Physics D: Applied Physics, vol. 4, pp. 938-950, jul 1971.

[22] L. Gao, W. Mei, M. Kleeberger, H. Peng, and J. Fottner, "Modeling and Discretization of Hydraulic Actuated Telescopic Boom System in Port-Hamiltonian Formulation," in Proceedings of the 9th International Conference on Simulation and Modeling Methodologies, Technologies and Applications, pp. 69-79, SCITEPRESS - Science and Technology Publications, 2019.

[23] W. Acuna-Bravo, E. Canuto, S. Malan, D. Colombo, M. Forestello, and R. Morselli, "Fine and simplified dynamic modelling of complex hydraulic systems," in Proceedings of the American Control Conference, pp. 5480-5485, 2009

[24] E. Franco, F. Rodriguez Y Baena, and A. Astolfi, "Robust Dynamic State Feedback for Underactuated Systems with Linearly Parameterized Disturbances," International Journal of Robust and Nonlinear Control, vol. 30, pp. 4112-4128, jul 2020. 